FORMATION Formation emploi

Revue française de sciences sociales

136 | octobre-décembre 2016

Pêle-mêle

\title{
Entraîneur de football professionnel : itinéraire d'un joueur gâté ?
}

Trainer of professional football : route of a spoiled player?

Profi-Fußballtrainer : Laufbahn eines verwöhnten Spielers?

Entrenador de fútbol profesional : itinerario de un jugador mimado?

Jean Bréhon, Hugo Juskowiak et Loïc Sallé

\section{OpenEdition}

Journals

Édition électronique

URL : http://journals.openedition.org/formationemploi/4907

DOI : 10.4000/formationemploi.4907

ISSN : 2107-0946

Éditeur

La Documentation française

Édition imprimée

Date de publication : 31 décembre 2016

Pagination : 55-77

ISSN : 0759-6340

\section{Référence électronique}

Jean Bréhon, Hugo Juskowiak et Loïc Sallé, « Entraîneur de football professionnel : itinéraire d'un joueur gâté ? », Formation emploi [En ligne], 136 | octobre-décembre 2016, mis en ligne le 31 décembre 2018, consulté le 30 octobre 2020. URL : http://journals.openedition.org/formationemploi/4907 ; DOI : https://doi.org/10.4000/formationemploi.4907 


\section{Entraîneur de football professionnel : itinéraire d'un joueur gâté?}

JEAN BRÉHON

Maître de conférences en STAPS (Sciences et techniques des activités physiques et sportives) au sein de l'Atelier SHERPAS (Sociologie, Histoire, Education, Représentations, Pratiques et Activités Sportives) - URePSSS, EA 7369 (Unité de Recherche Pluridisciplinaire Sport, Santé, Société),

université d'Artois

HugO JuSKOWIAK Maître de conférences en STAPS (Sciences et techniques des activités physiques et sportives) au sein de l'Atelier SHERPAS (Sociologie, Histoire, Education, Représentations, Pratiques et Activités sportives) - URePSSS, EA 7369, université d'Artois

Loïc SALLÉ

Maître de conférences en STAPS (Sciences et techniques des activités physiques et sportives) à l'Atelier SHERPAS (Sociologie, Histoire, Education, Représentations, Pratiques et Activités sportives) - URePSSS, EA 7369, université Lille 2

Résumé

Entraîneur de football professionnel : itinéraire d'un joueur gâté ?

Si l'accès à la profession d'entraîneur de football de l'élite (ligue 1 et ligue 2) est réglementé par des formations et titres sélectifs dont la Fédération française de football est seule dépositaire, existe-t-il une figure idéale-typique dominante des techniciens reçus au Brevet d'entraîneur professionnel ? Dans cet article, nous proposons d'analyser les trajectoires des stagiaires reçus au diplôme d'entraîneur. À cet effet, nous nous intéressons particulièrement aux caractéristiques statistiques des lauréats, depuis 2002, pour montrer que l'accès à la formation et au diplôme, la position occupée à l'entrée sur le marché sont étroitement liés à des avantages cumulés par les stagiaires au cours de leur parcours professionnel de joueur et de technicien.

Mots-clés : métier du sport, cheminement professionnel, formation professionnelle continue, accès à la FPC, relation formation-emploi

Abstract

Trainer of professional football: route of a spoiled player?

If the access to the occupation of trainer of football of the elite (league 1 and league 2) is regulated by selective formations and titles of which the French federation of football is only agent, does there exist a dominant ideal-typical figure of the technicians received 
with the Patent of professional trainer? We propose, in this article, to analyze the trajectories of the trainees received with the diploma for the occupation of trainer. For this purpose, we are interested particularly in the characteristics of the award-winnings, since 2002, to show that the access to the formation and the diploma, the position occupied at the entry on the market is closely related to advantages cumulated by the trainees during their career of player and technician.

Keywords: occupation in sport, occupational paths, continuing vocational training, access to CVT, relationship between training and employment

Journal of Economic Literature: L 83, J 44, J 24

Traduction : Auteurs.

Faut-il avoir été un footballeur de haut niveau pour être un entraîneur de haut niveau ? Pour les spécialistes de la discipline, répondre à cette question renvoie régulièrement à la mobilisation d'exemples et/ou de contre-exemples. L'exemple de sportifs à la carrière jalonnée de nombreux titres nationaux et internationaux et qui, seulement quelques mois après avoir " raccroché " les crampons, connaissent le succès en tant qu'entrâ̂neur. Le contre-exemple de certains techniciens, issus du monde amateur et n'ayant jamais pratiqué au haut niveau en tant que joueur, qui deviennent puis qui durent en qualité d'entraîneur professionnel de football. Complexe et biaisée par de nombreuses représentations alimentées par le traitement médiatique dont le football fait l'objet, cette question peut être objectivée au moins à deux niveaux.

D'un point de vue réglementaire, d'abord. Selon l'article R221-9 du code du sport, la qualité "d'entraîneur de haut niveau " s'obtient par l'inscription sur la liste correspondante arrêtée par le ministre des Sports, sur proposition de la fédération délégataire compétente, après avis du directeur technique national. La qualité d'entraîneur renvoie donc vers les statuts et les règlements de la Fédération française de football (FFF,) qui stipulent que pour exercer en qualité d'entraîneur " principal » sur les bancs de Ligue 1 et de Ligue 2, le technicien doit être titulaire du Brevet d'entraîneur professionnel de football $(\mathrm{BEPF})^{1}$. L'accès au métier est ainsi réglementé, soumis à une architecture des formations respectant les différents niveaux du football et répond à des logiques de reconnaissance des certifications professionnelles (RNCP).

Du point de vue des fondements de l'activité professionnelle, ensuite. La fonction d'entraîneur consiste à mettre en place une démarche systématique visant à fournir aux sportifs des moyens pour développer leur potentiel et réaliser leurs objectifs (Lemieux, Mignon, 2006). Le cœur du métier reposerait ainsi, notamment, sur une activité

1. Ce titre opére depuis 2012. Il remplace le diplôme d'entraineur professionnel de football (DEPF) proposé entre 1991 et 2012. 
de planification pour préparer les matchs et de prise de décisions instantanées pour répondre aux situations rencontrées en cours de jeu (Bertrand, 2009).

Si le métier d'entraîneur s'est complexifié et diversifié, notamment depuis la fin du vingtième siècle (Grün, 2011), aujourd'hui, il exige un haut niveau de compétences et de connaissances dont il est a priori difficile d'évaluer le poids de la carrière sportive dans leur acquisition, leur mobilisation et leur reconnaissance.

Or, quelques données statistiques interpellent. Les vingt derniers championnats de football professionnel (Ligue 1) organisés depuis la saison 1994/1995 ont été remportés par dix clubs et quinze entraîneurs différents. En resserrant la focale sur le profil de ces derniers, on note que onze entraîneurs sur les quinze ont préalablement embrassé une carrière de footballeur de haut niveau et qu'a contrario, quatre techniciens n'ont pas fréquenté les terrains de la première division. Cette tendance se confirme en élargissant l'analyse au profil de tous les techniciens du football de l'élite sur la même période : près de $90 \%$ d'anciens joueurs professionnels se retrouvent ainsi à la tête des équipes professionnelles de Ligue 1 et de Ligue 2 (Juskowiak, Sallé, Bréhon, 2015).

\section{Schéma 1 : Organisation du football français}

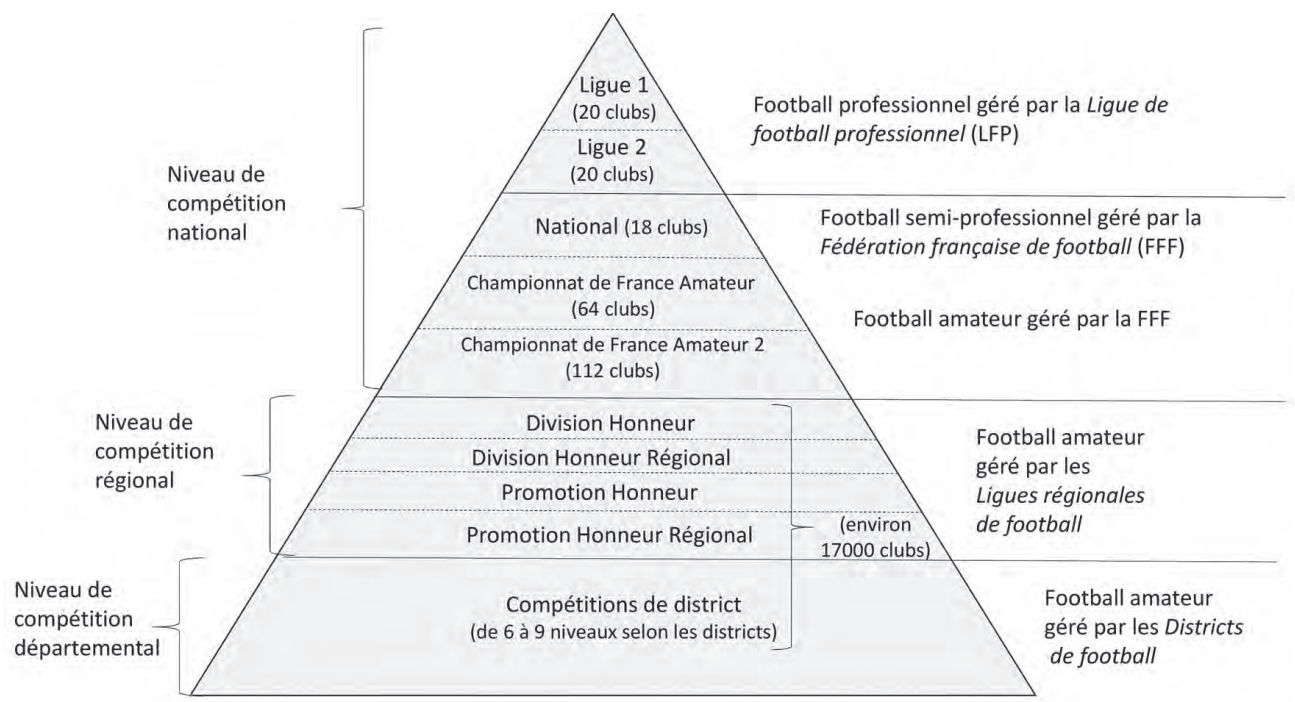

Source : Auteurs. 
Dès lors, comment expliquer cette tendance? Expérience accrue du terrain? Connaissance fine du milieu ? Maîtrise poussée des aspects technique, tactique, athlétique et psychologique d'une équipe ? Légitimité et crédibilité assurées auprès des joueurs ? Image et rayonnement positifs auprès des dirigeants de clubs ? Tous ces arguments (et d'autres) sont régulièrement convoqués par les acteurs et les commentateurs du football pour justifier les recrutements et les évictions, de plus en plus réguliers, d'entrâneurs soumis à des conditions de travail particulièrement instables. Mais il y a plus.

Si le football de haut niveau a fait l'objet de très nombreux travaux ${ }^{2}$, la profession d'entraîneur de football et son évolution ont essentiellement été travaillées dans une perspective historique.

On sait notamment que le droit d'entrée sur ce marché du travail sportif est intimement lié, depuis les années 1990, à un processus de professionnalisation marqué par la rationalisation des dispositifs de recrutement et la clarification des conditions d'accès au métier (Grün, 2004). On sait également que ces réformes constituent "le point d'orgue d'une phase visant à protéger l'accès de la profession en privilégiant les membres de la famille du football, à savoir les anciens joueurs professionnels " (Grün, ibid.). Pour autant, existe-t-il un profil-type dominant qui produit et préserve cette logique de "l'entresoi » (Pinçon, Pinçon-Charlot, 2007) ? Plus largement, les caractéristiques « des élites » (Leferme-Falguières, Van Renterghem, 2000) parvenant à trouver une place sur le marché du football professionnel sont-elles communes ou peut-on distinguer certaines singularités ? Par ailleurs, si les changements contemporains relatifs à l'architecture des formations et des titres requis permettent d'identifier des critères de sélection objectifs, la "licence " ne peut parfois suffire au "mandat " et celui-ci, dans l'univers du football professionnel, peut aussi s'acquérir sans l'obtention de la licence (Hughes, 1963). En effet, contrairement aux règlements affichés par l'institution, $22 \%$ des entraîneurs principaux, depuis $1991^{3}$, ne sont pas titulaires du BEPF et contournent les règlements fédéraux en intégrant, dans le staff technique, un adjoint (le prête-nom) qui dispose des qualifications requises pour exercer à haut niveaút.

Là encore, quelques particularités interrogent sur l'accès à cette activité professionnelle. A quoi sert le BEPF ? Le diplôme est-il une simple certification pour un technicien déjà en activité ou une licence pour embrasser une nouvelle carrière?

2. Citons, parmi d'autres: Wahl, 2002 ; Faure, Suaud, 1999 ; Dietschy, 2010, sur l'évolution et la structuration du football professionnel français ; Grün, 2011, sur la transformation et le fonctionnement du marché du travail des entraîneurs.

3. Diplôme d'entrâ̂neur professionnel de football, Décret n 91-270 du 7 mars 1991.

4. Notons d'ailleurs qu'avant 2015 , les règlements de la Fédération française de football prévoyaient une certaine souplesse de la règle en accordant, notamment, un délai au club pour l'obtention, par les techniciens en poste, des diplômes nécessaires à la participation aux compétitions. 
À ce titre, l'histoire et la sociologie des professions (Becker, 1963 ; Abbott, 1988 ; Menger, 2009) fournissent un cadre d'analyse fécond pour mettre en relation les caractéristiques spécifiques de ce groupe professionnel avec les atouts dont dispose chacun de ses membres.

Plus précisément, le modèle des " avantages cumulatifs " (qualité, compétences, réussite, voire simple facteur aléatoire), initialement proposé par Merton (1968), cherche à expliquer les écarts de notoriété professionnelle à partir de mécanismes internes à l'offre. L'analyse des temps de la formation et du début de carrière peut révéler, pour des professionnels aux caractéristiques interindividuelles proches, des avantages initiaux (même minimes). En s'accumulant, ceux-ci peuvent provoquer des différences importantes de distribution des bénéfices, ainsi que des réputations construites suivant les caractéristiques propres au groupe professionnel et à la dynamique des appariements sélectifs disponibles.

Ce modèle explique, en partie, la diversité du groupe professionnel rencontré, "soumis à des changements continus, caractérisés à la fois par des contours évolutifs et une hétérogénéité interne " (Demazière, Gadéa, 2009). Aussi, ces avantages, plus ou moins prononcés, peuvent se décliner différemment suivant l'analyse des trajectoires des individus concernés. C'est pourquoi, pour Menger (2009), l'étude des professions incertaines nécessite de se pencher plus particulièrement sur les formations initiales et les premiers pas d'une carrière pour observer les transferts opérant à ces étapes décisives de constitution des réputations.

Pour les entraineurs de football, la formation au BEPF constitue un observatoire privilégié pour identifier les profils de ceux qui accèdent (ou pas) au monde professionnel. Et ce, non seulement par l'examen des critères de sélection qui pèsent sur les candidats postulant à la formation, l'analyse des caractéristiques (sportives et sociologiques) des stagiaires formés, mais aussi par l'étude des stratégies de contrôle adoptées par les formateurs pour réguler l'entrée dans cet univers professionnel.

S'intéresser aux processus de formation et à la part des "avantages initiaux " dans l'entrée sur le marché de l'emploi éclaire ainsi autrement les trajectoires et dynamiques de carrière de cette figure sportive peu travaillée scientifiquement. Les enjeux essentiels de ce texte sont de comprendre comment s'organise la formation des futurs techniciens, de caractériser les entrants sur le marché professionnel des entraîneurs et d'identifier les traits de cette figure produite par les instances du football. ${ }^{5}$ À ce titre, les avantages cumulés à l'entrée et au cours de la formation permettent de mieux appréhender la construction de cette catégorie professionnelle. Peu abordée sous cet angle, l'étude de ce groupe, par la mobilisation d'un cadre théorique multiréférencé et combiné (Paquette, 2007), permet d'approcher différemment les étapes de fabrication des professionnels en formation et de questionner autrement l'entrée sur le marché de l'emploi .

5. Les auteurs souhaitent ici remercier Oumaya Hidri-Neys, membre de l'équipe SHERPAS, pour les précieux conseils apportés. 


\section{Encadré 1 : Méthodologie de la recherche}

Les résultats proposés ici sont issus d'une recherche conventionnée (1) et reposent sur quatre méthodologies articulées : l'étude des modules d'enseignement du BEPF (Brevet d'entraîneur professionnel de football), complétée par des observations in situ de trois sessions de formation organisées à Clairefontaine (lieu de formation et de rassemblement des équipes de France de football) par la Direction technique nationale de football ; l'analyse qualitative de 32 entretiens semi-directifs menés auprès de l'ensemble des stagiaires et formateurs au BEPF ( $n=15$, session 2012-2014) et d'entraîneurs professionnels en poste ( $n=17$, ligue 1 et 2$)$; et enfin le traitement statistique de 90 fiches professionnelles reconstituées (données saisies et traitées, $n=2000$ ) de l'ensemble des entraîneurs formés (actifs ou non), depuis 2002, par la Fédération française de football.

Les entretiens réalisés auprès des techniciens s'organisent autour de quatre axes : le parcours professionnel de l'enquêté, son entrée dans la carrière, ses modes d'exercice de l'activité, les conditions pour durer dans le métier. Pour chacun d'entre eux, l'angle de questionnement privilégié repose, entre autres, sur l'impact de la formation sur ces différents segments de l'activité professionnelle.

Les critères choisis répondent à la mise en évidence de moments clés qui construisent la carrière des professionnels (Becker, 1963 ; Hughes, 1963). Dans une perspective d'entretien compréhensif (Kaufmann, 2011), chacun de ces items est alors tour à tour intégré dans une « spirale de l'analyse » (Blanchet, Gotman, 2010) qui s'organise autour du croisement des positions objectives des individus, des points de vue indigènes et des pratiques réelles des enquêtés.

Pour réduire davantage les éventuels biais méthodologiques, les discours des différents acteurs sont complétés par une série d'observations outillées sur le terrain (20 séances d'entraînement ou moments de séances) et par la recomposition des fiches biographiques retenues qui représentent l'ensemble du groupe des entraîneurs stagiaires formés depuis 2002.

En mobilisant les sources disponibles (2), la carrière de joueur, l'activité à l'entrée en formation et à la sortie, le statut (professionnel ou amateur), le poste occupé au sein du staff technique, le nombre de clubs fréquentés, les réussites obtenues (championnat et coupes nationales) sont ainsi mis à jour pour chaque entraîneur identifié. Le panorama présenté situe donc relativement bien la figure du technicien formé, au moins pour la période la plus récente.

(1) Convention de recherche collaborative, 2012-2016. Entrer, exercer, durer, évoluer dans le métier d'entraîneur de football: analyse des trajectoires, carrières et activités de coach. Union nationale des entraîneurs et cadres techniques professionnels du football.

(2) Pour l'ensemble des tableaux proposés dans cet article, les sources mobilisées sont issues des Archives de la Fédération française de football, des archives de I'UNECATEF (Union nationale des entraîneurs et cadres techniques professionnels du football), ainsi que des données recueillies sur Footdatabase, plateforme spécialisée dans le recensement des acteurs du football de haut niveau. 
Dans une première partie, nous montrons en quoi la catégorie des entraîneurs de haut niveau est intimement liée à la figure de l'ancien joueur professionnel expérimenté. L'avantage d'une reconversion assumée, voire valorisée par l'ensemble des acteurs engagés dans ce processus, semble offrir une meilleure lisibilité sur le marché et assurer une maîtrise précoce des différentes facettes du métier. Or, si ce trait caractéristique du groupe observé se retrouve dans les discours des acteurs, il se révèle étonnamment modeste dans la construction du parcours de formation du stagiaire et de son début de carrière, en tout cas si on le considère isolément.

Dans une deuxième partie, nous montrons que cet avantage initial jugé nécessaire mais pas suffisant, est complété d'une activité de terrain déjà bien réelle et pour laquelle les savoirs pratiques et les expériences acquises sont soumis à une double contrainte : celle de leur efficacité, d'une part, et de la position professionnelle déjà acquise, d'autre part, qui participent à la construction de la réputation du coach (Menger, op. cit.). Le niveau, la durée d'engagement et la réussite dans les premières expériences d'entraîneur permettent ainsi de consolider, au sein de la formation, une position professionnelle préexistante. Ils distinguent entre eux les stagiaires et futurs candidats aux postes : "L'avantage procuré, tôt dans la carrière, par des qualités repérées, peut être faible, mais il suffit qu'il y ait à chaque épreuve de comparaison compétitive, une différence perceptible, petite ou grande, pour attirer les investissements et les paris des acteurs du système." (Menger, ibid).

Dans une dernière partie, nous mettons donc en évidence comment un professionnel ayant acquis, de façon précoce, une réputation ou ayant connu un succès repéré dans son monde professionnel bénéficiera de ressources et d'investissements cumulés à la fois dans la formation suivie et dans le déroulement de sa «nouvelle » carrière qui débute.

\section{L'expérience de joueur professionnel, un avantage pour entrer en formation?}

Dans l'univers professionnel des entraîneurs, être issu du monde des joueurs professionnels confère un avantage certain pour entrer dans la formation d'entraîneur. Si cet avantage peut, en grande partie, garantir une place en formation initiale, pour autant, il ne présage en rien de la réussite en formation et de l'entrée sur le marché.

Si le diplôme d'entraîneur professionnel de football est actuellement présenté comme "une formation complète qui permet aussi bien à ceux qui viennent du très haut niveau qu'à ceux qui viennent de la base de pouvoir y accéder et aussi de pouvoir réussir l'examen " (Directeur technique national, 2010), les résultats statistiques nuancent le propos indigène. Les candidats issus du football amateur sont, en réalité, isolés, et représentent seulement $15 \%$ des techniciens retenus et reçus. De 2002 à 2014, les stagiaires formés et titulaires du diplôme sont, en très grande majorité, d'anciens joueurs professionnels jouissant d'une carrière relativement longue $(85 \%)$. 
Tableau 1. Eléments de carrière des stagiaires reçus au BEPF (2002-2014)

\begin{tabular}{|l|c|c|}
\hline \multicolumn{1}{|c|}{ Statut } & \multicolumn{2}{|c|}{ Carrière de joueur } \\
\hline Joueur Amateur & \multicolumn{2}{|c|}{73 soit 15\% soit 85\% } \\
\hline Joueur Professionnel & \multicolumn{2}{|c|}{ Durée de la carrière de joueur (en saison) } \\
\hline & 13 soit 15\% & \multicolumn{2}{|c|}{$15 \%$} \\
\hline Amateur & 4 soit 5\% & \multirow{2}{*}{ 12\% } \\
\hline Contrat pro (1-3 saisons) & 3 soit 2\% & \multirow{2}{*}{$73 \%$} \\
\hline Carrière courte (4-5) & 4 soit 5\% & \\
\hline Carrière moyenne (6-8) & 38 soit 42\% & \\
\hline Carrière longue (9-14) & 28 soit 31\% & \\
\hline Carrière très longue (15 et +) & 10,86 & \\
\hline Moyenne & 20 & \\
\hline
\end{tabular}

Exemple de lecture : 12,1 \% des stagiaires reçus au BEPF (Brevet d'entraîneur professionnel de football) sur la période d'étude présentent une carrière de joueur qualifiée de " courte » ou « moyenne ».

Les pourcentages sont arrondis à l'unité la plus proche et ne font pas apparaitre de dixième.

Source : Auteurs.

Si l'on retient comme indice la durée moyenne de carrière d'un joueur de Ligue 1 ou de Ligue $2^{6}, 73 \%$ d'entre eux ont exercé durablement l'activité de footballeur (plus de neuf saisons), voire témoignent d'une longévité remarquable (plus de quinze saisons pour $31 \%$ des entraîneurs recensés). Cette première caractéristique est par ailleurs stable dans le temps, puisqu'une seule session de formation, depuis 2002, comptabilise un peu plus de $20 \%$ de stagiaires issus du monde amateur.

Les plus hautes formations fédérales permettant d'exercer au sein de l'élite du football sont donc principalement réservées aux anciens joueurs pro. Cette situation s'explique par un processus historique de structuration de la profession d'entraîneur dans lequel la DTN française (1971-1975, 1988-1991, 2008-2012) aura joué un rôle militant. Les travaux de Laurent Grün (op. cit.) montrent notamment que les principaux responsables de la DTN de football ont œuvré, tour à tour, pour créer diplômes et formations permettant de distinguer l'éducateur de football de l'éducateur d'Etat, avec pour conviction la reconnaissance des capitaux sportifs et symboliques (Bourdieu, 1992) comme principale variable de recrutement. En 1975, le Directeur technique national soulignait déjà que "l'essentiel des connaissances et du bagage de l'entraîneur s'acquiert pendant la carrière de joueur, par l'entrâेnement, par les matchs, par les discussions" (repris de Grün, op. cit.).

Aujourd'hui, l'argument de la connaissance du milieu est toujours avancé par les formateurs : "C'est comme pour nous, si l'on nous donnait à conduire une formule 1, on se planterait (...) au premier virage, on se planterait, c'est sûr. Le joueur de haut niveau a, lui, cette capacité à maîtriser plus rapidement toutes les facettes du métier d'entraîneur. " (Propos tenus par A., 64 ans, ancien entraîneur professionnel de club, responsable de la formation BEPF)

6. Soit 6 à 8 saisons, source retenue : Union nationale des footballeurs professionnels. 
Cette posture institutionnelle se concrétise par les pré-requis et les conditions d'accès au BEPF, fixés par les instances du football ainsi que par les différents aménagements prévus pour le joueur de haut niveau ${ }^{7}$. Un ancien joueur de niveau international, justifiant de dix sélections en équipe de France ou d'au moins 150 matchs en tant que joueur professionnel en ligue 1, peut en effet candidater directement à l'entrée en formation. C'est le signe d'une logique d'entre-soi et de cooptation (Pinçon, Pinçon-Charlot 2007) d'une profession obéissant finalement aux usages élitaires (Leferme-Falguières, Van Renterghem, 2000), au moins du point de vue de la régulation de ses conditions d'entrée. Ce que confirment certains entretiens. À l'instar de J., 57 ans, ancien joueur professionnel division 1, stagiaire 2002-2004 :

"Je n'avais pas prévu de passer la formation (...) Les délais de dépôt du dossier étaient d'ailleurs écoulés. Et puis un soir, A. (un formateur) me téléphone et me dit d'envoyer ma candidature. Je lui dis qu'il est trop tard. Il insiste. Et puis voilà quoi (...) Là-bas? à Clairefontaine, avec $S$ et $R$. on était toujours au fond. Qu'est-ce qu'on a rigolé. Mais eux, ils n'étaient pas trop embêtés. Enfin, là ce que je raconte, c'est off. S. on ne lui a jamais demandé de passer en pédagogie. Je me souviens un jour, S; anime une situation. Ouh la la! Ce n'était pas bon. On ne comprenait pas. Et T. (le formateur) s'est retourné, a jonglé avec le ballon comme si de rien nétait. "

La constitution " d'un public cible s'inscrit ainsi dans un processus dynamique justifié, aux yeux des acteurs, par la fascination de l'expérience de haut-niveau et la volonté manifeste des dirigeants de participer à la reconversion des anciens joueurs"(Grün, op. cit.). L'histoire de la professionnalisation des entraîneurs explique ici, en grande partie, le trait caractéristique soulevé pour des licenciés d'une profession prestigieuse qui en exerce étroitement le contrôle.

D’ailleurs, la composition du pool de formateurs/évaluateurs reflète également cette situation. Depuis 1991 et la création du DEPF, Gérard Houllier, Raymond Domenech, René Girard ou encore Francis Smerecki, figures du football français, ont été désignés par la DTN comme responsables du diplôme, chargés de concevoir et de superviser chaque session de formation. De Philippe Bergeroo à Guy Lacombe, en passant par Pierre Mankowski, nombreux sont les entraîneurs expérimentés, reconnus pour leur longévité dans la carrière, à intégrer également l'équipe de formation de Clairefontaine.

Pour les candidats n'ayant pas eu une carrière de joueur significative, il faut montrer, en qualité de technicien, une implication réelle et régulière dans le football de l'élite : "Ils ont besoin de cela pour la reconnaissance du milieu ", nous confie le responsable national de la formation des entraîneurs en 2002, "plus que les autres qui n'ont pas besoin de justifier " Ainsi, "être ou avoir été entraîneur assistant de l'équipe première d'un club autorisé à utiliser des joueurs professionnels durant cinq saisons sportives" ou "être titulaire du Brevet d'entraîneur formateur de football et exercer ou avoir exercé, à ce titre, dans un centre agréé "haut-niveau",

7. Conformément à la fiche pédagogique 2014/2016 du BEPF produite par l'Institut de formation du football (IFF). 
durant cinq saisons sportives " permet de postuler à la formation auprès de la DTN (fiche RNCP).

Aussi, pour les entraîneurs issus du football amateur, les exigences et/ou les étapes sont encore plus nombreuses. Cela passe soit par les succès sportifs : l'accession (et le maintien) à la tête de son équipe au plus haut niveau des championnats amateurs nationaux ("être ou avoir été entraîneur de l'équipe première d'un club non professionnel opérant au niveau national durant cinq saisons sportives»), soit "être titulaire du diplôme immédiatement inférieur à celui $d u B E P F$ » (le Diplôme d'entraîneur supérieur) qui s'obtient en gravissant les différents échelons, après un cursus de formation de onze années. Ainsi, même si les portes de la profession s'entrouvrent aux éducateurs amateurs, ces derniers portent « le fardeau de leur trajectoire» (Pilet, 2003).

Ils doivent impérativement se construire une légitimité que la carrière de footballeur ne leur confère pas. L’entrée dans la profession se caractérise donc, pour eux, par des débuts précoces comme entrâneur, en parallèle de leur activité de joueur, contrairement aux joueurs professionnels. 80 \% des entraîneurs, anciens joueurs amateurs, entrânaient déjà avant leur trente ans, et ont encadré une équipe dix ans au moins avant de se voir confier un effectif professionnel.

La carrière de B. illustre bien cette situation singulière. Joueur amateur de niveau fédéral, il a entraîné pendant plus de dix ans dans le circuit régional chez les jeunes et les seniors, avant d'entrer dans la formation professionnelle, obtenant, au passage, la montée de son club en National.

À l'inverse, après avoir participé à plus de 600 matchs en professionnel (20 saisons au plus haut niveau) et avoir porté le maillot bleu à plusieurs reprises, M. prend sa retraite en 2003. Inexpérimenté dans l'encadrement du football, il devient néanmoins stagiaire BEPF en 2004 et obtient le diplôme, en 2006, au terme de la formation. Il occupe son premier poste de technicien en Ligue 1 dès l'année suivante.

Les pré-requis exigés illustrent ainsi clairement le choix d'une figure aux caractéristiques sélectives, imposée et souhaitée par le monde du football lui-même : l'ancien joueur professionnel expérimenté. Celui-ci bénéficie donc d'un avantage certain, assumé et revendiqué. Suffit-il, pour autant, pour entrer dans le groupe professionnel des entraîneurs certifiés et débuter dans la carrière?

\section{- Activité du stagiaire et mise à distance de la formation, un avantage cumulé non négligeable?}

La caractérisation statistique de l'effectif des 90 BEPF diplômés permet de dresser deux autres constats majeurs et de discuter des avantages cumulés par tout ou une partie de la population étudiée. 
Le premier constat est celui de la succession des temps de carrière : la formation au BEPF, en tendance, ne se superpose pas à la carrière de joueur professionnel. Dans 89 cas, soit presque la totalité, l'entrée en formation s'effectue une fois que la carrière de joueur est achevée. Pour autant, cette uniformité apparente ne doit pas masquer les disparités qui peuvent exister entre les différents individus : si la durée moyenne entre la fin de carrière de joueur et l'entrée au BEPF est de neuf ans environ, la distribution moyenne est de six ans et les extrêmes s'étendent de 0 à 23 ans d'intervalle. L'anticipation plus ou moins grande de la reconversion ${ }^{8}$, l'existence de dispositifs de formation accélérée pour certaines populations ou l'évolution personnelle dans le métier de technicien permettent sans doute de comprendre un peu mieux ces variations.

Tableau 2 : Activité des entraîneurs stagiaires à l'entrée en formation

\begin{tabular}{|c|c|c|c|c|c|}
\hline $\begin{array}{l}\text { Activité du technicien à } \\
\text { l'entrée au BEPF }\end{array}$ & \multicolumn{2}{|c|}{$\begin{array}{l}\text { Nombre de } \\
\text { techniciens }\end{array}$} & $\begin{array}{l}\text { Actif } \\
\text { joueur }\end{array}$ & $\begin{array}{l}\% \text { sur les } 90 \text { BEPF } \\
\text { formés-reçus }\end{array}$ & $\begin{array}{l}\% \text { sur les } 69 \text { BEPF } \\
\text { formés-reçus actifs }\end{array}$ \\
\hline $\begin{array}{l}\text { Entraîneur Principal de Ligue } 1 \\
\text { ou de Ligue } 2\end{array}$ & 21 & \multirow{7}{*}{$\begin{array}{l}77 \% \text { d'actifs } \\
(n=69)\end{array}$} & 0 & $24 \%$ & $30 \%$ \\
\hline $\begin{array}{l}\text { Membre d'un staff de Ligue } 1 \\
\text { ou de Ligue } 2\end{array}$ & 20 & & 0 & $22 \%$ & $29 \%$ \\
\hline $\begin{array}{l}\text { Formateur dans un club à } \\
\text { statut professionnel }\end{array}$ & 10 & & 0 & $11 \%$ & $15 \%$ \\
\hline $\begin{array}{l}\text { Sous-total Football } \\
\text { professionnel }\end{array}$ & 51 & & 0 & $57 \%$ & $74 \%$ \\
\hline $\begin{array}{l}\text { Entraîneur principal d'une } \\
\text { équipe amateur }\end{array}$ & 15 & & 0 & $17 \%$ & $22 \%$ \\
\hline Membre d'un staff amateur & 3 & & 0 & $3 \%$ & $4 \%$ \\
\hline Sous-total amateur & 18 & & 0 & $20 \%$ & $26 \%$ \\
\hline Inactif & 21 & $\begin{array}{l}23 \% \text { d'inactifs } \\
\quad(n=21)\end{array}$ & 1 & $23 \%$ & \\
\hline
\end{tabular}

Exemple de lecture : les 21 techniciens évoluant en qualité d'entraîneur principal de Ligue 1 ou de Ligue 2 représentent $21 \%$ des stagiaires BEPF (Brevet d'entraîneur professionnel de football) formés-reçus au BEPF et $30 \%$ des formés-reçus actifs. Les pourcentages sont arrondis à l'unité la plus proche et ne font pas apparaître de dixième.

Source : Auteurs.

8. Sur ce point, voir également les travaux de Papin (1987). 
Le second constat est relatif à l'activité - ou à l'absence d'activité - professionnelle des stagiaires avant leur entrée en formation. Partant de l'hypothèse que l'accès à certains marchés - et donc l'entrée dans une activité professionnelle - est conditionné à l'obtention d'un titre ou d'un diplôme particulier, véritable droit d'entrée (Hughes, 1996), il aurait été légitime de s'attendre à ce que le BEPF, fréquemment présenté comme le moyen d'accès privilégié à la profession d'entraîneur professionnel de football, constitue un réel déclencheur du passage de l'inactivité à l'activité.

Or, les données statistiques démontrent que plus des trois quarts des techniciens (77\%) sont déjà en activité (professionnelle ou amateur) lorsqu'ils entrent en formation et que près des trois quarts de ces actifs $(74 \%)$ le sont dans le milieu du football professionnel. A contrario, parmi les 21 techniciens inactifs qui entrent en formation, 17 sont d'anciens joueurs professionnels. En ce sens, le BEPF semble davantage constituer un outil d'occupation ou de solidification dans l'espace professionnel qu'un véritable droit d'entrée sur le marché des entraîneurs professionnels de football.

Quant aux techniciens acceptés en formation, issus du monde amateur, ils présentent un profil particulier. Ils appartiennent en effet au football amateur de haut niveau (CFA2 à National) et occupent majoritairement un poste d'entraîneur principal. Se pose, dès lors, la question des raisons qui conduisent à recruter en formation des " insiders " (Becker, 1963), des techniciens d'ores et déjà en poste pour la plupart.

L'un des éléments structurels que nous pourrions avancer tient à l'organisation pyramidale de la formation des éducateurs français. Excepté les dérogations accordées aux anciens joueurs de haut niveau, les conditions d'entrée dans les différents diplômes d'encadrement du football associent une solide expérience de terrain à la détention du diplôme de rang juste inférieur. La première condition d'accès à l'entrée au DES JEPS mention football (Diplôme d'Etat supérieur de la jeunesse, de l'éducation populaire et du sport, immédiatement inférieur au BEPF, de niveau II, inscrit au RNCP) est " de justifier d'une expérience d'entraîneur de football d'une équipe de niveau régional senior pendant au moins six cent heures au cours des trois dernières saisons sportives" (Fiche récapitulative du DES JEPS mention football, Institut de Formation du Football, 2014). 
Tableau 3 : Volumes horaires et contenus de la formation BEPF

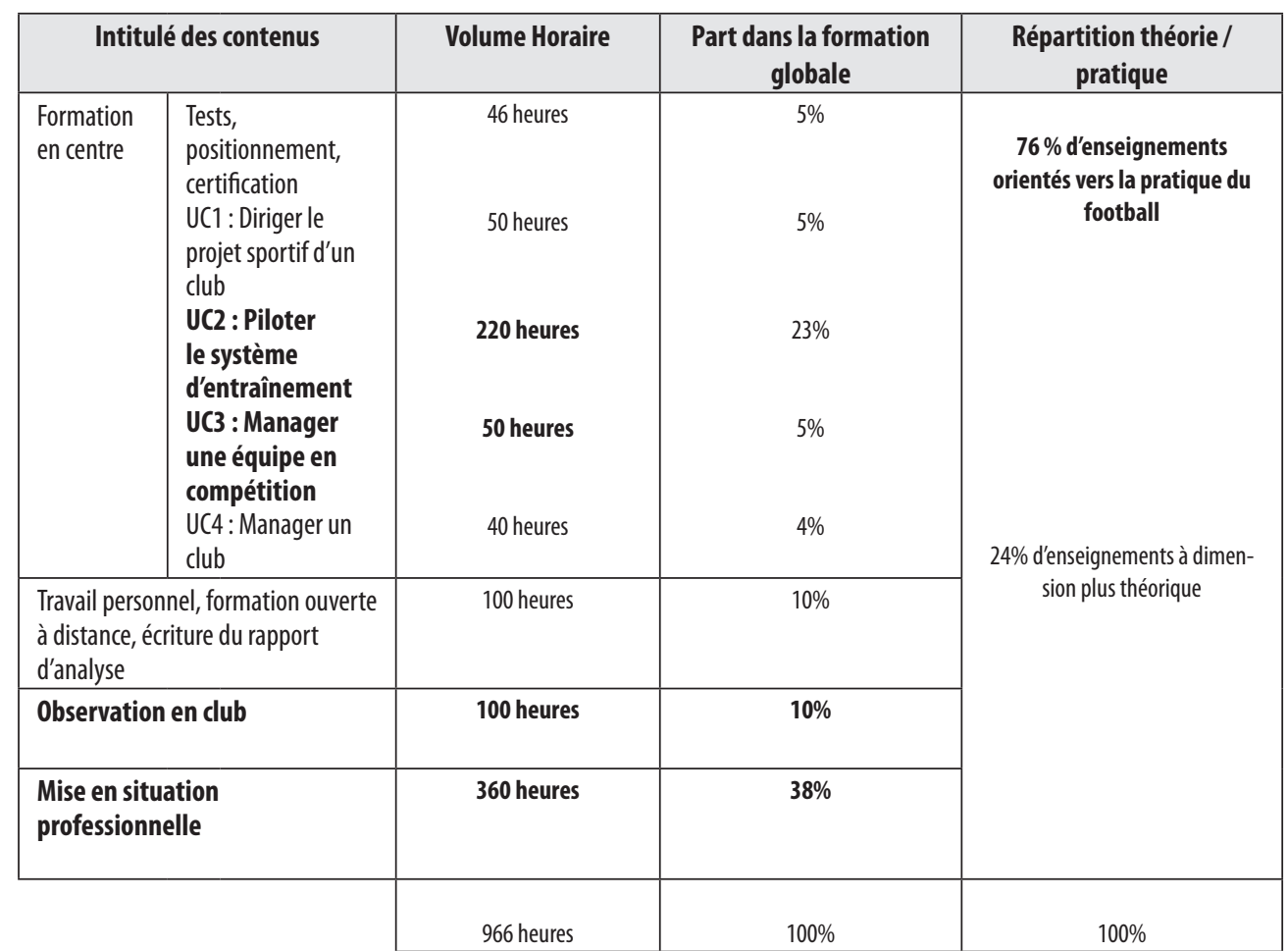

Note : Les enseignements à dimension « pratique » apparaissent en gras dans le tableau.

Exemple de lecture : sur un total de 966 heures de formation, $76 \%$ des enseignements sont orientés vers la pratique du football, soit 720 heures consacrées à l'entraînement, au management de l'équipe, à l'observation en club et à la mise en situation professionnelle.

Les pourcentages sont arrondis à l'unité la plus proche et ne font pas apparaitre de dixième.

Source : Auteurs.

En même temps qu'il valorise, à l'entrée, les " hommes de terrain ", le BEPF renforce également l'acquisition de compétences pratiques en cours de formation. Les contenus proposés aux stagiaires font en effet la part belle aux savoirs pratiques. La fiche RNCP du BEPF prévoit, en effet, 966 heures de formation, réparties sur deux saisons, dont les trois-quarts sont articulés autour de savoirs pratiques : 360 heures en situation professionnelle (stage d'intervention club), 100 heures d'observation en club, 220 heures dédiées au pilotage du système d'entraînement de l'équipe première de football, dans un club autorisé à utiliser des joueurs professionnels ou d'une sélection nationale, et 50 heures centrées sur le management d'une équipe en compétition?.

9. En comparaison, le DEPF proposait un volume horaire total de 630 heures, distribuées autour d'enseignements théoriques $(200 \mathrm{~h})$, pratiques $(190 \mathrm{~h})$ et de mises en situation de stage $(240 \mathrm{~h})$ : avec la création du BEPF, on note une augmentation significative des heures dédiées au management des clubs et des temps 
Là encore, les discours des formateurs entrent en résonnance avec les attentes fixées par les maquettes de formation :

"On essaie de leur apporter l'expérience que l'on a pu avoir, mais après, c'est comme toujours dans toutes les formations, c'est l'utilisation sur le terrain, en direct, dans tous les domaines, quand on est confronté à la situation. On a montré le mode d'emploi, mais maintenant il faut qu'il l'utilise et c'est là toute la vraie difficulté. " (Propos de A., 64 ans, ancien joueur professionnel et entraîneur professionnel de club, responsable de la formation BEPF)

Les observations menées aux cours des différentes sessions de travail, les entretiens réalisés auprès des trois responsables du diplôme renvoient l'idée d'une formation articulée autour de deux axes principaux : un terrain d'expérimentations et de réflexions autour des animations de jeu disponibles pour l'entraîneur de club et la découverte des outils nécessaires à la gestion du football professionnel. Les options retenues par les techniciens dans les clubs le seront sur la base des contextes, particularités locales et expériences vécues.

La plupart des stagiaires produisent également un discours sur la formation qui valorise son versant pratique : "la gestion du terrain ", pour le formé, est au cœur de la définition du métier (Demazière, 2005) :

"Après, tu as des cours d'arbitrage... pourquoi pas ... Tu as cours de gestion et comptabilitélo. Tout ça, c'est bien, mais aujourd'hui, il faut dire que nous, ce que l'on veut, c'est le BEPF. Je ne vais pas faire les contrats et je ne vais pas faire la comptabilité du club. Donc, on est un peu plus réfractaires à ces cours si tu veux. On y va, on écoute, on sait que c'est une formation globale, mais tu es plus réfractaire à ces choses-là quà la pédagogie. Quand on est sur le terrain avec P. ou avec F, voilà, c'est ça que l'on aime, c'est le cour du métier. " (Propos tenus par B., 43 ans, ancien joueur professionnel division 2, à la recherche d'un emploi, stagiaire en formation, session 2012-2014).

Pour autant, et même si ce volet est largement valorisé par les stagiaires, ces derniers relativisent l'importance de leur apprentissage initial. Tenu pour essentiel dans l'entrée sur le marché, l'enseignement reçu est perçu comme secondaire, l'activité du " technicien en train de faire » dans les clubs étant jugée prioritaire par rapport à l'activité « du stagiaire en train de se faire » en formation.

"Tu sais, ce que l'on voit en cours, c'est important, c'est vrai. Parfois, c'est un plus, mais pas souvent. Parce que ce que l'on nous propose ici, on le connaît déjà plus ou moins. Alors on nous donne des modèles, mais qu'on ne peut pas transposer dans nos clubs. Ce n'est pas comme cela que ça marche. Oui, c'est utile pour avoir le diplôme et pour se faire remarquer après, mais pour le reste, c'est sur le terrain, avec nos joueurs, que l'on apprend; c'est l'expérience vécue qui est essentielle " (Propos de C., entraîneur en National. Stagiaire, session 2012-2014).

de stage proposés aux stagiaires.

10. Ces contenus spécifiques font partie de deux modules (UC3 et 4) du BEPF. 
Plus encore, le formateur incarne contradictoirement " un modèle et un repoussoir, le détenteur de compétences désirables et celui dont l'étudiant doit apprendre à récuser l'autorité " (Menger, op. cit). Ce type de relation pédagogique déjà étudié (injonction paradoxale) provoque l'effet d'une compétition entre le maître et l'élève, d'autant plus forte que les emplois à la clé les placent, parfois, en situation de concurrence (Merton, 1988). Ce qui est précisément le cas pour la profession d'entraîneur, certains formateurs quittant la DTN pour réintégrer un club professionnel ${ }^{11}$.

Ces formes de résistance à la formation peuvent aussi être considérées, par le formateur et le stagiaire lui-même, comme un avantage non négligeable pour la suite. L'insoumission ou plutôt la mise à distance des savoirs et pratiques enseignés est aussi considérée comme une forme d'originalité et de singularité utiles pour se frayer un chemin sur un marché pour lequel le formateur peut être tout à la fois candidat concurrent aux postes à pourvoir ou soutien précieux pour l'obtention d'un contrat :

"J'essaie toujours d'être critique. C'est utile parce que ça nous force à réfléchir. Les cours, c'est vraiment bien, mais il faut faire la part des choses. Moi, ça fait dix ans que je roule ma bosse sur les terrains des clubs professionnels. Ma formation, elle est là. Et puis, surtout, ça peut faire la différence entre nous. On ne sait jamais. Pour obtenir le diplôme et aussi pour la suite. Parfois, c'est la DTN qu'on appelle, quand il y a des postes qui se libèrent. " (D., 42 ans, ancien joueur professionnel division 1, à la recherche d'un emploi. Stagiaire, session 2012-2014)

Le cumul emploi/formation et appropriation sélective des contenus dispensés forme donc un second trait avantageux pour le futur professionnel. La période de l'apprentissage réussie peut être finalement perçue comme la socialisation anticipée du futur professionnel et cela, de deux manières complémentaires : l'identité professionnelle se construit par et contre autrui et l'apprenti s'installe dans le métier à partir de ses premières expériences vécues. L'avantage pour le candidat qui s'inscrit dans cette logique serait d'autant plus important que les taux de réussite à l'examen sont élevés. En effet, sur la période analysée, plus de $90 \%$ des stagiaires entrant en formation sont titulaires du brevet à la sortie du cycle de formation. Les différences interpersonnelles se situeraient donc bien ailleurs.

\section{Position dans l'espace professionnel et capital réputationnel, un avantage décisif ?}

Le modèle de l'avantage cumulatif permet d'analyser, pour des individus aux caractéristiques proches, les inégalités de réussite comme "le produit d'une dynamique de divergence croissante des trajectoires à partir d'une situation initiale de quasi-égalité des chances" (Menger, 2009). Si cet avantage est d'abord très légèrement favorable, il peut provoquer

11. Même s'ils sont peu nombreux, citons, par exemple, le cas de René Girard, aujourd'hui entraîneur du Football Club Nantes Atlantique. 
des différences considérables. Dans cette troisième partie, nous cherchons à mesurer l'impact du BEPF sur le début de carrière des stagiaires sortants. Pour ce faire, nous comparons notamment les trajectoires respectives d'individus dotés d'une formation et de ressources de formation quasi-équivalentes.

Tableau 4 : Impact du BEPF sur les carrières des techniciens

\begin{tabular}{|c|c|c|c|c|c|c|c|}
\hline & \multicolumn{3}{|c|}{ Activité à l'entrée } & \multicolumn{3}{|c|}{ Activité à la sortie } & \multirow{2}{*}{$\begin{array}{c}\text { Evolution } \\
\text { entre } \\
\text { l'entrée et } \\
\text { la sortie de } \\
\text { formation }\end{array}$} \\
\hline & $\begin{array}{l}\text { Nombre de } \\
\text { techniciens }\end{array}$ & $\begin{array}{l}\% \text { sur les } \\
90 \text { BEPF } \\
\text { formés- } \\
\text { reçus }\end{array}$ & $\begin{array}{l}\% \text { sur les } \\
69 \text { BEPF } \\
\text { formés- } \\
\text { reçus } \\
\text { actifs }\end{array}$ & $\begin{array}{l}\text { Nombre de } \\
\text { techniciens }\end{array}$ & $\begin{array}{c}\% \text { sur les } \\
90 \text { BEPF } \\
\text { formés- } \\
\text { reçus }\end{array}$ & $\begin{array}{l}\% \text { sur les } \\
74 \text { BEPF } \\
\text { formés- } \\
\text { reçus actifs }\end{array}$ & \\
\hline $\begin{array}{l}\text { Entraineur } \\
\text { principal de } \\
\text { Ligue } 1 \text { ou de } \\
\text { ligue } 2\end{array}$ & 21 & $24 \%$ & $30 \%$ & 37 & $41 \%$ & $50 \%$ & $+16(18 \%)$ \\
\hline $\begin{array}{l}\text { Membre d'un } \\
\text { staff de Ligue } 1 \\
\text { ou Ligue } 2\end{array}$ & 20 & $22 \%$ & $29 \%$ & 12 & $13 \%$ & $16 \%$ & $-8(9 \%)$ \\
\hline $\begin{array}{l}\text { Formateur dans } \\
\text { un club à statut } \\
\text { professionnel }\end{array}$ & 10 & $11 \%$ & $15 \%$ & 10 & $11 \%$ & $14 \%$ & 1 \\
\hline $\begin{array}{l}\text { Sous total } \\
\text { Football } \\
\text { professionnel }\end{array}$ & 51 & $57 \%$ & $74 \%$ & 59 & $65 \%$ & $80 \%$ & $+8(9 \%)$ \\
\hline $\begin{array}{l}\text { Entraîneur } \\
\text { principal d'une } \\
\text { équipe à statut } \\
\text { amateur }\end{array}$ & 15 & $17 \%$ & $22 \%$ & 14 & $16 \%$ & $19 \%$ & $-1(1 \%)$ \\
\hline $\begin{array}{l}\text { Membre d'un } \\
\text { staff amateur }\end{array}$ & 3 & $3 \%$ & $4 \%$ & 1 & $1 \%$ & $1 \%$ & $-2(2 \%)$ \\
\hline $\begin{array}{l}\text { Sous-total } \\
\text { amateur }\end{array}$ & 18 & $20 \%$ & $26 \%$ & 15 & $17 \%$ & $20 \%$ & $-3(3 \%)$ \\
\hline
\end{tabular}

Exemple de lecture : sur les 90 stagiaires formés-reçus au BEPF (Brevet d'entraîneur professionnel de football), 21 exercent une activité d'entraîneur principal de Ligue 1 ou de Ligue 2. À la sortie de la formation, ils sont 37 à exercer cette fonction au plus haut niveau, soit 50 \% des stagiaires reçus-formés actifs. On constate donc une augmentation de $18 \%$ de l'activité entre l'entrée au BEPF et l'obtention du diplôme. Les pourcentages sont arrondis à l'unité la plus proche et ne font pas apparaître de dixième.

Source : Auteurs. 
Premier constat, à l'issue des deux années de formation, les diplômés du BEPF sont, statistiquement, à la fois moins exposés à l'inactivité (- $5 \%$ ) et moins présents dans le monde amateur $(-3 \%)$. De manière concomitante, ils sont plus nombreux à exercer leur activité au sein du football professionnel $(+8 \%)$. En ce sens, et conformément à ce que nous avancions comme hypothèse précédemment, le BEPF apparaît comme un outil de stabilisation, voire d'accélération des carrières. Cela semble d'autant plus vrai que parmi les seize techniciens répertoriés comme inactifs, neuf sont en reprise d'activité moins de deux saisons après l'obtention du $\mathrm{BEPF}^{12}$. Au final, si l'on comptabilise les stagiaires diplômés actifs à l'issue de la formation, ils sont $82 \%$ à exercer une activité et ce taux à $\mathrm{N}+2$ après l'obtention du BEPF grimpe à 92 \% (83 actifs sur 90 entraîneurs).

Quantitativement, le BEPF apparaît donc comme un outil efficace dans la mise en activité ou le maintien en activité des entraîneurs. Pouvons-nous dresser le même type de constat du point de vue qualitatif ? Sur les 74 entraîneurs en activité recensés à la sortie du diplôme, 59 (soit 80 \%) le sont dans le monde professionnel et 15 (20\%) dans le monde amateur. Dès lors, le fléchage du BEPF par la DTN comme passeport pour l'encadrement technique d'une équipe à statut professionnel devient plus fragile. Il le devient encore plus si l'on examine la répartition des postes obtenus dans le milieu professionnel : parmi les 59 actifs concernés, 14 exercent en qualité d'entraîneur professionnel en L1 (24 \% des actifs), 23 en tant qu'entraîneur principal en L2 (39 \% des actifs), 7 sont entraîneurs adjoints en L1/L2 (12\% des actifs), les 15 techniciens restants (25\%) occupant des postes à la formation ou dans les staffs techniques des clubs professionnels. Au final, sur l'ensemble des 90 techniciens titulaires du BEPF, seuls 14 exercent en tant qu'entraîneur principal en Ligue $1(16 \%)$ et 23 en Ligue $2(25 \%)$, ce qui conduit à un taux d'adéquation entre le diplôme et le poste de $41 \%$ seulement.

Le BEPF se définit donc bien comme un levier privilégié d'entrée dans le monde professionnel, mais sans aucune garantie d'exercice au poste le plus prisé. L'obtention du précieux sésame consolide vraisemblablement une position sur le marché des entraîneurs professionnels. Le marché de l'emploi, quant à lui, s'organiserait suivant des règles différentes :

"Autre point qui a sans doute aussi une influence, au niveau professionnel, je pense que d'avoir une carrière de joueur aide quand même pour passer tout de suite au niveau professionnel sans passer par l'étape CFA, national et autre et obtenir des résultats. Ça, c'est la deuxième chose et puis après, il y a surtout les opportunités à saisir avec un réseau, une reconnaissance des clubs, une communication, entre guillemets, nécessaires... (E., 62 ans, ancien joueur professionnel, ancien entraîneur professionnel de club, formateur et entraîneur national)

Tous les techniciens sortant de la formation au BEPF n'accèdent donc pas directement ou n'accèderont pas aux postes les plus élevés. Certains d'entre eux y parviennent cependant. En effet, le tableau 5 révèle une augmentation de 16 points de la catégorie des entraîneurs

12. Soit $23 \%$ des reçus au BEPF. Voir Supra tableau 2. 
de L1/L2, entre l'entrée et la sortie du diplôme. Pour autant, cette donnée mathématique " brute " ne représente que la somme des trajectoires professionnelles ascendantes et descendantes. Pour une analyse plus fine, il faut se rapprocher des acteurs, de leurs parcours personnels et professionnels (Demazière, Gadéa, 2009), pour se rendre compte que les diplômés BEPF connaissent, en réalité, 19 trajectoires ascendantes.

Tableau 5 : Caractérisation des 19 formés-reçus BEPF accédant directement à des postes d'entraîneur principal Ligue 1- Ligue 2

\begin{tabular}{|c|c|}
\hline Postes occupés par les stagiaires à l'entrée en formation & Nombre de stagiaires \\
\hline Entraîneur principal L1/L2 & $0(0 \%)$ \\
\hline Membre du staff L1/L2 & $1(5 \%)$ \\
\hline Formateur club Professionnel & $6(31 \%)$ \\
\hline Entraîneur principal amateur & $5(27 \%)$ \\
\hline Membre du staff amateur & $0(0 \%)$ \\
\hline Inactif & $7(37 \%)$ \\
\hline
\end{tabular}

Exemple de lecture : sur les 19 formés-reçus BEPF (Brevet d'entraîneur professionnel de football) accédant directement à un poste d'entraineur principal de l'élite, cinq (soit $5 \%$ ) occupaient, à l'entrée en formation, un poste d'entraîneur principal dans un club amateur.

Source : Auteurs.

En termes de carrières professionnelles, le tableau 5 permet d'illustrer le fait que la formation au BEPF ne profite pas de la même manière à tous les participants. L'étude systématique des parcours de ces coachs (sept formateurs, cinq amateurs, sept inactifs) laisse à penser que certains avantages (carrière de joueur, vécu de joueur), insignifiants lorsqu'ils sont appréhendés séparément, se combinent pendant le temps de la formation et permettent d'infléchir les carrières des prétendants. Reconnues par les formateurs, les stagiaires et progressivement intégrées par les candidats eux-mêmes, ces synergies donnent à voir trois types de trajectoires professionnelles après l'obtention du diplôme.

Premier cas de figure identifié, celui de l'entraineur principal amateur ayant joué et officié aux plus bas niveaux de pratique et qui gravit les échelons du football fédéral avec son équipe et accède aux niveaux supérieurs. Ou encore celui du technicien qui réalise des exploits, en Coupe de France notamment. Dans le premier cas, le responsable technique de l'équipe concernée, pour répondre aux obligations d'encadrement des compétitions fixées par le statut des éducateurs de football de la Fédération Française de Football, doit disposer, à moyen terme, du niveau de formation et du diplôme associés au niveau sportif du championnat dans lequel son équipe évolue. L'usage se conforme ici à la règle, l'entraineur amateur se professionnalise, volontairement ou par obligation, au rythme de sa progression dans l'élite sportive (Gasparini, Pierre, 2008). L'obtention du BEPF lui permet d'être en règle avec l'institution. Il poursuit sa trajectoire ascendante le plus souvent avec l'équipe dont il a la charge depuis quelques saisons. L'entraîneur amateur peut aussi bénéficier de "l'effet Coupe " comme d'une aubaine lui permettant de mettre en lumière ses compétences supposées (Sorignet, 2004 ; Bertrand, 2009 ; Lemieux, Mignon, op. cit). 
"Là donc, je suis à Châtellerault, on fait des bonnes saisons et tout, on va loin en Coupe de France, c'est un club qui était renommé, au niveau national, pas professionnel, puisquion ne l'était plus, mais au niveau national. Et là G. minntègre dans son équipe de formateur à la DTN. Un bon parcours en Coupe, ça aide pour la carrière! " (F., 55 ans, entraîneur professionnel retraité division 2, stagiaire BEPF en 1994)

La valorisation de l'épreuve opposant clubs amateurs et professionnels est telle que le technicien peut profiter de la situation et jouer de son capital réputationnel pour se faire connaitre et reconnaître aux yeux du grand public, des agents et des présidents agissant sur le marché (Lemieux, Mignon, ibid) :

"Dans le milieu du foot, tarrives à déceler les trucs, táarrives à démasquer, etc., mais les dirigeants ne sont pas des connaisseurs de foot et ils sont passionnés, certes, mais pas forcément des connaisseurs et du coup, ils peuvent se laisser amadouer. Voilà, on voit souvent, quand une équipe n'est pas très bien, les dirigeants qui se tournent vers des coachs à poigne pour resserrer tout ça. Donc, les mecs qui n’ont pas de postes, ils jouent là-dessus pour être à poigne, ou inversement sil faut un mec plus tourné sur le jeu.» (G., 50 ans, entraîneur professionnel Ligue 1, stagiaire BEPF en 2010)

Deuxième cas de figure, le formateur, installé au poste dans le club professionnel et ancien joueur de bon niveau (Ligue 2 en général ; les six recensés jouissent tous d'une activité de plus de sept saisons au club), bénéficie des circonstances du moment ; en effet, la crise de résultats de l'équipe professionnelle amène les dirigeants à démettre de ses fonctions le titulaire et à privilégier la «solution interne» (Sallé, Bréhon, Juskowiak, 2016). Ou encore, "la promotion interne" est retenue par les dirigeants pour assurer la continuité du travail réalisé par le prédécesseur démissionnaire ou arrivant en fin de contrat. Là encore, les interactions et stratégies d'acteurs semblent réelles (Lemieux, Mignon, op. cit).

Troisième cas de figure rencontré, l'entraîneur professionnel fait une pause dans sa carrière "pour souffler et recharger les batteries" (propos tenus par un entraîneur professionnel, stagiaire BEPF en 2012) et retrouve un poste plus ou moins rapidement en ligue 1 ou 2. Ou encore, le technicien, ancien joueur de très haut niveau (Ligue 1 voire international) frâchement diplômé qui accède directement à l'élite du football français ; ici, les bénéfices sportif, symbolique, communicationnel et réputationnel sembleraient jouer un rôle non négligeable (Grün, op. cit.). Mais cela ne touche qu'une faible partie de la population étudiée. Ils sont 19 dans cette situation sur les 90 répertoriés, sans compter le nombre de postes disponibles à haut niveau depuis dix ans. En effet, chaque saison, 43 postes d'entraîneurs principaux sont redistribués (ou non) aux demandeurs toujours plus nombreux. Les cumuls des avantages identifiés plus haut peuvent expliquer ces entrées ou retours dans la carrière "par le haut».

Pour ces différentes configurations, il convient enfin de souligner le rôle des acteurs clés du recrutement, agents sportifs et présidents de clubs (Jouvenet, Demazière, 2013 ; Pilet, 2003 ; Schotté, 2016). Le réseau constitue aussi un ressort essentiel pour expliquer l'ob- 
tention (ou non) des postes convoités. C'est aussi par l'attention que lui prêtent les autres que le technicien trouve ou non place dans le football de haut niveau :

"Pour trouver un poste, c'est sûr, tout est important: la formation, les expériences de terrain, le projet de jeu que tu portes, ta réussite, l'image que tu renvoies..., mais ce qui est sûr, c'est que si tu ne séduis pas le président que tu as en face de toi, si ton réseau ne t’aide pas à forcer quelques portes, alors tu es mort. » (H., 66 ans, ancien entraîneur professionnel)

\section{Conclusion}

Peut-on dégager une figure dominante des acteurs retenus pour entrer en formation au Brevet d'entraîneur professionnel de football ? Existe-t-il un type-idéal de lauréat qui décroche directement un poste dans les clubs professionnels après l'obtention du diplôme ? Voici les deux principales interrogations formulées dans cet article. D’emblée, deux tendances lourdes apparaissent : il faut justifier d'un vécu de joueur de football et faire état d'une activité d'encadrement d'équipe déjà conséquente pour attirer l'attention des formateurs et " entrer " au Centre technique national Fernand Sastre de Clairefontaine.

Mais nos résultats montrent que cette figure dominante ici dévoilée s'efface rapidement devant la diversité des profils d'acteurs investis dans la formation. En effet, tous n'ont pas le même vécu de joueur (de l'amateur de haut niveau à l'ancien international) et tous n'encadrent pas dans les mêmes divisions (du niveau Championnat de France amateur à la Ligue 1).

Ces variations, qui peuvent sembler presque infimes, à l'origine, lorsqu'elles sont observées isolement, vont se cumuler lors du temps de formation pour devenir significatives et construire des profils identitaires différents "pour soi" mais aussi "pour autrui " (formateurs, autres stagiaires, intervenants) (Dubar, 2002). Cette identité professionnelle se construit et/ou se renforce, à la fois par le stagiaire dans ses réalisations dans et hors des temps de formation, mais également par les formateurs qui viennent "étiqueter " (Becker, op. cit.) tel ou tel individu comme potentiel entraîneur de haut niveau. Dès lors, nous comprenons mieux pourquoi des individus dotés d'une formation et de ressources quasi-équivalentes en arrivent à occuper des positions différentes dans l'espace de l'encadrement professionnel, autrement dit, pourquoi tous les techniciens sortant de la formation n'accèdent pas directement ou n'accèderont pas aux postes les plus élevés.

Cependant, certains y parviennent et trois profils se dégagent. N'étant pas d'anciens joueurs de haut niveau, les premiers comblent "le fardeau de leur trajectoire " (Pilet, 2003) par une performance en Coupe de France ou des montées sportives successives. Les seconds sont des "insiders" (Becker, ibid.) ; anciens professionnels sans avoir joué les premiers rôles, avant l'entrée en formation, ils occupent des postes dans les staffs des clubs professionnels, qu'ils parviennent à consolider une fois le diplôme obtenu. La troisième catégorie regroupe d'anciens joueurs de très bon niveau (Ligue 1, voire des 
internationaux) qui cumulent un vécu de technicien dans les plus hautes divisions. En reprise d'activité ou directement après l'obtention du diplôme, ces profils proposant un fort capital sportif, symbolique, communicationnel et réputationnel (Grün, op. cit.) sont susceptibles d'occuper les postes les plus convoités. La focale particulière de la formation et de l'accès à l'emploi utilisée ici pour observer les entraîneurs professionnels de football révèle, en réalité, une grande variété dans les profils, les positions et les trajectoires des prétendants aux plus hauts niveaux de l'encadrement du football français. Elle permet, in fine, de discuter du rôle relatif joué par la formation au BEPF à l'entrée sur le marché du travail ; l'obtention du diplôme pouvant ici être considérée comme un outil de consolidation dans l'espace professionnel.

Cette hétérogénéité " des techniciens entrants " autorise finalement à penser la catégorie des entraîneurs non comme un "ensemble protégé, fermé ou codifié, mais davantage comme un processus évolutif, vulnérable, ouvert, instable" (Demazière, Gadéa, op. cit.) qui, comme d'autres, fluctue dans sa composition, la nature des tâches accomplies par les individus qui le composent, la conception de leur rôle ou encore les anticipations de carrière (Hughes, 1958). Ces disparités illustrent bien la nécessité, pour mieux saisir les réalités, de multiplier les approches et d'élargir les cadres d'analyse (Mendras, Oberti, 2000 ; Mucchielli, 2005).

\section{- Bibliographie}

Abbott A. (1988), The System of the Professions. An Essay of the Division of Expert Labour, Chicago University Press, 452 p.

Becker H. (1963), Outsiders: Studies in the Sociology of Deviance, New York, Free Press, 247 p.

Bertrand J. (2009), "Entre "feeling” et objectivations : les manières d'entraîner dans un centre de formation au football professionnel ", Sciences de la Société, n 77, Tousouse, Presses universitaires du Mirail, pp .65-79.

Blanchet A., Gotman A. (2010), L'enquête et ses méthodes, Paris, Armand Colin, 128 p.

Bourdieu P. (1992), Les règles de l'art : genèse et structure du champ littéraire, Paris, Seuil, 480 p.

Demazière D., Gadéa C. (2009), Sociologie des groupes professionnels. Acquis récents et nouveaux défis, Paris, La Découverte, 456 p.

Demazière D. (2005), "Au cœur du métier de facteur : "sa” tournée ", Ethnologie française, Vol. 35, pp 129-136.

Dietschy P. (2010), Histoire du football, Paris, Perrin, 620 p. 
Dubar C. (2002), La socialisation, construction des identités sociales et professionnelles, Paris, Armand Colin, 278 p.

Faure J.-M., Suaud C. (1999), Le football professionnel à la française, Presses Universitaires de France, 260 p.

Gasparini W., Pierre J. (2008), «Vendre et se vendre. Dispositions et compétences des vendeurs d'articles de sport ", STAPS n 80, pp. 43-55.

Grün L. (2004), "La difficile émergence de la profession d'entraîneur de football en France (1890-1950)", STAPS n 63, pp. 45-62.

Grün L. (2011), Entraîneur de football : histoire d'une profession de 1890 à nos jours, Thèse de doctorat STAPS, université Claude Bernard Lyon 1, 1082 p.

Hughes E.C. (1958), Men and their work, Glencoe, The Free Press, 2e éd, 184 p.

Hughes E.C. (1963), "Professions ", Daedalus, n 92, traduit par J.-M. Chapoulie sous le titre «Les professions établies », E.C. Hughes éd., 1996, Le Regard sociologique, Paris, EHESS, pp. 107-122.

Juskowiak H., Sallé L., Bréhon J. (2015), « Devenir entraîneur pro de football : entre formation et valorisation d'acquis ", Revue juridique et économique du sport, 159, pp. 40-45.

Jouvenet M., Demazière D. (2013), “The market work of football agents and the manifold valorizations of prof”, Economic Sociology, the European electronic newsletter, vol. 15, pp. 29-40.

Leferme-Falguières F., Van Renterghem V. (2000), " Le concept d'élites. Approches historiographiques et méthodologiques ", Hypothèses Publications de la Sorbonne, pp. 55-67.

Lemieux C., Mignon P. (2006), Etre entraîneur de haut niveau : Sociologie d'un groupe professionnel entre marché du travail fermé et marché du travail concurrentiel, Rapport de recherche INSEP, $171 \mathrm{p}$.

Kaufmann J.-C. (2011), L'entretien compréhensif. L'enquête et ses méthodes, Paris, Armand Colin, $128 \mathrm{p}$.

Menger P.-M. (2009), Le travail créateur. S’accomplir dans l'incertain, Paris, GallimardSeuil, coll. « Hautes Études », 670 p.

Merton R.K. (1968), “The Matthew effect in science”, Science, 159 (3810), pp. 56-63.

Merton R.K. (1988), “The Matthew effect in science, II: Cumulative advantage and the symbolism of intellectual property", ISIS, 79 (299), pp. 606-623.

Mendras H., Oberti M. (2000), Le sociologue et son terrain. Trente recherches exemplaires, Paris, Armand Colin, 304 p. 
Mucchielli A. (2005), "Le développement des méthodes qualitatives et l'approche constructiviste des phénomènes humains ", in Royer C., Baribeau C., Recherches qualitatives et production de savoirs, Recherches qualitatives Hors-série "Les Actes ", 1, pp. 41-60.

Papin B. (1987), Conversion et reconversion des élites sportives : approche socio-historique de la gymnastique artistique et sportive, Paris, L'Harmattan, 286 p.

Paquette D. (2007), « Le rôle du cadre de référence théorique dans une recherche monographique constructiviste ", Recherches qualitatives, volume 27, pp. 3-21.

Pilet E. (2003), Les trajectoires des entraîneurs dans le champ du football professionnel, mémoire pour le DEA de sciences sociales, université de Nantes, 2003, 311 p.

Pinçon M., Pinçon-Charlot M. (2007), Les ghettos du Gotha. Comment la bourgeoisie défend ses espaces, Paris, Seuil, coll. «Essais », 294 p.

Sallé L., Bréhon J., Juskowiak H. (2016), « Entraîneur de football : un travail d'équipe ? Décider, décider de déléguer, déléguer la décision ", in Dosseville F., Garncarzyk C. (dir.). Pratiques sportives et décision: une exploration de la complexité des contextes et des interactions, Caen, Presses Universitaires de Caen.

Schotté M. (2016), « Monter en première division. Trajectoires de notabilisation des présidents de clubs de football professionnel (1960-1999) ", Politix n 114, pp. 99-120.

Sorignet P.-E. (2004), « Un processus de recrutement sur un marché du travail artistique : le cas de l'audition en danse contemporaine ", Genèses n ${ }^{\circ}$ 4, pp. 64-88.

Wahl A. (2002), La balle au pied: histoire du football, Gallimard, 144 p. 


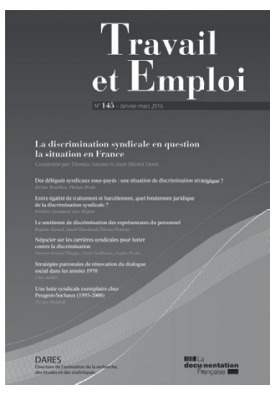

\section{TRAVAIL et EMPLOI}

\section{$\mathrm{n}^{\circ} 145$, janvier-mars 2016}

\section{La discrimination syndicale en question : la situation en France}

\section{INTRODUCTION}

La discrimination syndicale : une discrimination comme les autres ?

Enjeux et état des lieux

Thomas Amossé, Jean-Michel Denis

\section{ARTICLES}

Des délégués syndicaux sous-payés : une situation de discrimination stratégique?

Une analyse économétrique à partir de l'enquête REPONSE de 2010 Jérôme Bourdieu, Thomas Breda

Entre égalité de traitement et harcèlement, quel fondement juridique de la discrimination syndicale ?

État des lieux et analyse du contentieux entre 2012 et 2014

Frédéric Guiomard, Inès Meftah

Le sentiment de discrimination des représentants du personnel Une étude à partir des données statistiques et monographiques liées à l'enquête REPONSE

Baptiste Giraud, Amaël Marchand, Étienne Penissat

Négocier sur les carrières syndicales pour lutter contre les discriminations

Une appropriation sélective et minimaliste du droit

Vincent-Arnaud Chappe, Cécile Guillaume, Sophie Pochic

Stratégies patronales de rénovation du dialogue social dans les années 1970

Discrimination de l'action syndicale à la SNIAS de Toulouse Clair Juilliet

Une lutte syndicale exemplaire chez Peugeot-Sochaux (1995-2000)

La remise en cause d'un système discriminatoire Nicolas Hatzfeld

\section{NOTES DE LECTURE}

Christelle Avril, Les Aides à domicile. Un autre monde populaire Nehara Feldman

Anne Eydoux, Bernard Gomel (coord.), Apprendre (de l'échec) du RSA. La solidarité active en question Cécile Lefèvre

Philippe Brégeon, Parcours précaires. Enquête sur la jeunesse déqualifiée Vanessa Pinto

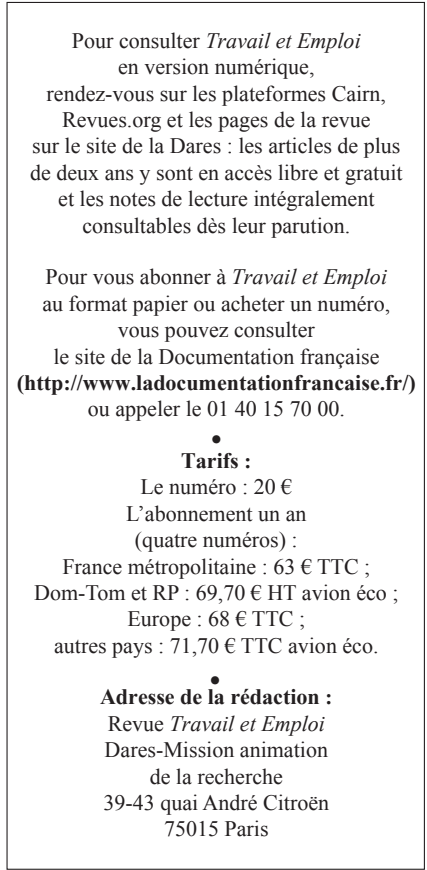

